\title{
An Analysis of the Motion Signal Distributions Emerging from Locomotion through a Natural Environment
}

\author{
Johannes M. Zanker ${ }^{1,2}$ \& Jochen Zeil ${ }^{2}$ \\ ${ }^{1}$ Department of Psychology, Royal Holloway, University of London, Egham, \\ Surrey TW20 0EX, England, j.zanker@rhul.ac.uk \\ 2 Centre for Visual Sciences, RSBS, The Australian National University, Canberra, \\ ACT 2601, Australia
}

\begin{abstract}
Some 50 years have passed since Gibson drew attention to the characteristic field of velocity vectors generated on the retina when an observer is moving through the three-dimensional world. Many theoretical, psychophysical, and physiological studies have demonstrated the use of such optic flowfields for a number of navigational tasks under laboratory conditions, but little is known about the actual flowfield structure under natural operating conditions. To study the motion information available to the visual system in the real world, we moved a panoramic imaging device outdoors on accurately defined paths and simulated a biologically inspired motion detector network to analyse the distribution of motion signals. We found that motion signals are sparsely distributed in space and that local directions can be ambiguous and noisy. Spatial or temporal integration would be required to retrieve reliable information on the local motion vectors. Nevertheless, a surprisingly simple algorithm can retrieve rather accurately the direction of heading from sparse and noisy motion signal maps without the need for such pooling. Our approach thus may help to assess the role of specific environmental and computational constraints in natural optic flow processing.
\end{abstract}

\section{Background}

Visual motion information is crucial for maintaining course, avoiding obstacles, estimating distance, and for segmenting complex scenes into discrete objects. Active locomotion generates large-scale retinal image motion that contains information both about observer movement - egomotion - and the three-dimensional layout of the world. The significance of optic flowfields has been recognised since Gibson (1950) illustrated the dynamic events in the image plane resulting from egomotion by sets of homogenously distributed velocity vectors. The actual structure of the twodimensional motion signal distributions experienced by the visual system under natural operating conditions, however, is not only determined by the pattern of locomotion, but also by the specific three-dimensional layout of the local environment and by the motion detection mechanism employed. To understand the design of the neural processing mechanisms underlying flowfield analysis, and in particular the coding strategies of motion sensitive neurones, we thus need to know more about the actual motion signal distributions under real-life conditions.

H.H. Bülthoff et al. (Eds.): Biologically Motivated Computer Vision 2002, Lecture Notes in Computer Science 2525, pp. 146-156, 2002

Springer-Verlag Berlin Heidelberg 2002 
An elaborate theoretical framework has been developed on how to extract egomotion parameters from flowfields (Longuet-Higgins and Prazdny, 1980; Heeger, 1987; Koenderink and Van Doorn, 1987; Dahmen et al., 1997). Most of these models assume implicitly that local motion signals are veridical, homogenously distributed, and carry true velocity information. Similarly, motion sensitive neurones in the visual systems of invertebrates and vertebrates that seem to be involved in flowfield processing (e.g., Hausen and Egelhaaf, 1989; Frost et al., 1990; Orban et al., 1992; Krapp and Hengstenberg, 1996) are usually investigated with coherently structured motion stimuli that densely cover large parts of the visual field. Franz \& Krapp (2000) and Dahmen et al. (2001) recently used simulations to assess the number and the distribution of motion signals that are necessary to estimate egomotion parameters. Their studies suggest that surprisingly few local and low fidelity flow measurements are needed, provided these measurements are taken at widely distributed locations throughout the panoramic visual field. So far, however, we do not know what kind of motion signal distributions visual systems are confronted with in a normal ecological and behavioural context.

To address this issue, we reconstructed a "view from the cockpit" of flying insects, which have become model systems for optic flow analysis because of their extraordinary behavioural repertoire and because the neural machinery underlying optic flow processing is well understood (Hausen and Egelhaaf, 1989; Eckert and Zeil, 2001). We moved a panoramic imaging device along accurately defined paths in outdoor locations containing a mixture of close and distant vegetation. We then analysed the image sequences which we recorded during these movements with a two-dimensional motion detector model (2DMD) consisting of an array of correlation-type detector pairs for horizontal and vertical motion components (Zanker et al., 1997). The analysis of movie sequences recorded on comparatively simple flight paths is intended to help us evaluate the image processing requirements involved in extracting egomotion information under realistic conditions. In particular, we can assess the density and spatial distribution of local motion signals normally available and the amount of noise that the visual system has to cope with under natural operating conditions. Eventually such an analysis will enable us to identify the image processing strategies the visual system could in principle employ. In order to cope with various sources of unreliable signals, obvious candidates for such processing strategies are for instance local gain control, spatial or temporal averaging, or the selection or combination of adequately tuned spatiotemporal channels. The focus of the present account is on a simple procedure to estimate the quality of motion signal maps by assessing the recovery of direction of heading for purely translatory movements.

\section{The approach}

A panoramic imaging device was mounted on a computer-controlled robotic gantry which allowed us to move it along accurately defined trajectories within a space of about $1 \mathrm{~m}^{3}$. Servo-controlled DC motors moved the camera along the three orthogonal axes with an accuracy of $0.1 \mathrm{~mm}$ (system components by Isel, Germany). The gantry was mounted on a trolley to be positioned in a variety of outdoor locations. For 
the present analysis we chose a location with grass, shrubs and bushes amongst large Eucalyptus trees, containing a mixture of close and distant objects, which would be representative of a typical habitat for flying insects.

A

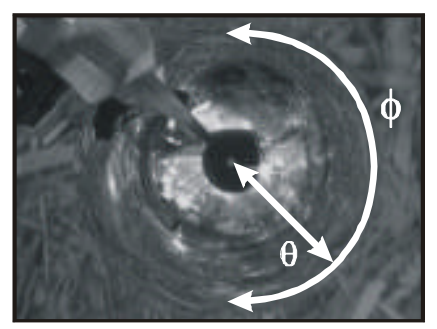

B

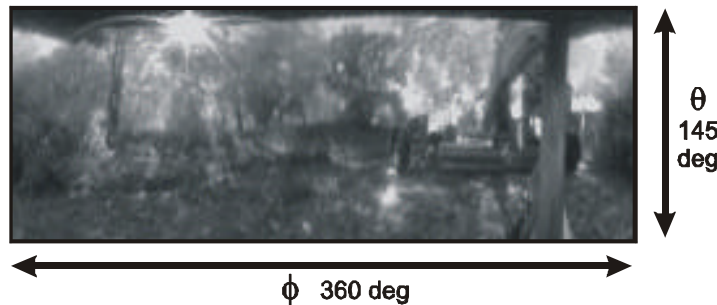

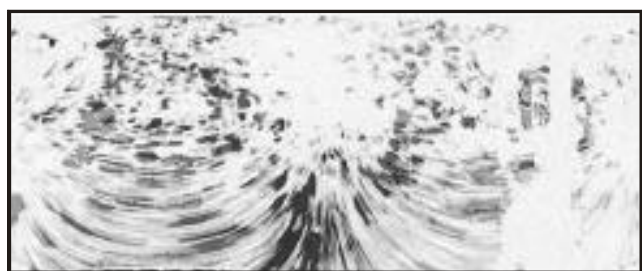

D

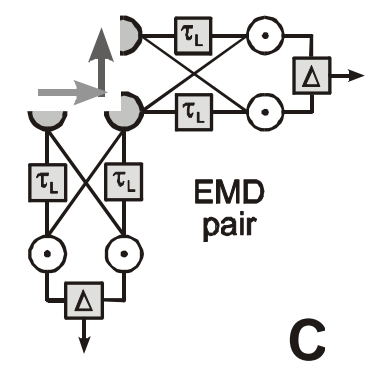

Fig. 1. A method to study flowfields in the real world. A video camera is used to capture panoramic images in polar coordinates $(\mathrm{A})$ which are converted into cartesian coordinates $(\mathrm{B}$; azimuth $\Phi$, elevation $\theta$ ). Image sequences were recorded while moving the camera through a natural scene and then used as input to a large array of motion detector pairs (one element sketched in C), to generate motion signal maps (D).

The panoramic imaging device consisted of a black and white video camera (Samsung BW-410CA) looking down onto a parabolic mirror which was optimised for constant spatial resolution (Chahl and Srinivasan, 1997). In the captured images the azimuth and elevation are represented in polar coordinates, as angular position and distance from the origin in the image centre, respectively (see figure 1A). Images were digitised with 8 bit greylevel resolution (Matrox Meteor framegrabber) and stored directly on a computer harddisk for off-line analysis. The image sequences were subsequently converted into cartesian coordinates, resulting in an image 450 pixels wide and 185 pixels high, which corresponds to a visual field size of $360^{\circ}$ of azimuth, $\Phi$, by $136^{\circ}$ of elevation, $\theta$, (figure 1B). In the default configuration, image sequences of 64 consecutive frames were taken at 25 frames/s during gantry speeds of $5 \mathrm{~cm} / \mathrm{s}$ and $10 \mathrm{~cm} / \mathrm{s}$. The apparatus also allows to grab single images could at sequences of predefined positions, in order to generate more complex trajectories. Image sequences were analysed with a two-dimensional, correlation-type motion detector model (2DMD) which has been used previously to identify the processing requirements faced by fiddler crabs in detecting and recognising species-specific 
movement signals (Zeil and Zanker, 1997), to study the characteristic patterns of image motion produced by wasps during their learning flights (Zeil et al., 1998), and to simulate a wide range of psychophysical phenomena (e.g., Zanker et al., 1997; Patzwahl and Zanker, 2000; Zanker, 2001). The basic building block is tha elementary motion detector (EMD) of the correlation type which has been shown in many behavioural and physiological studies to be a very good candidate for the biological implementation of motion processing (for review, see Reichardt, 1987; Borst and Egelhaaf, 1989). This EMD model is a representative of a variety of luminance based operators (Adelson and Bergen, 1985; Van Santen and Sperling, 1985; Watson and Ahumada, 1985), and other models for local motion detection (e.g., Srinivasan, 1990; Johnston et al., 1999) could be used without affecting the main conclusions we draw from our results. Orthogonal pairs of local EMDs that detect horizontal and vertical motion components (sketched in figure 1C) are used to build a two-dimensional network of detectors, which constitutes the 2DMD model.

In a simple implementation (figure 1C), each EMD receives input from two points of the spatially filtered stimulus patterns. The signals interact in a nonlinear way after some temporal filtering, to provide a directionally selective signal. Difference of Gaussians (DOGs) with balanced excitatory centre and inhibitory surround are used as bandpass filters in the input lines to exclude DC components from the input. The sampling distance between the two inputs, which is the fundamental spatial model parameter, was set to 2 pixels (approximately $1.6^{\circ}$ ) for the present study. To prevent aliasing, the diameter of the receptive field was set to about twice the value of the sampling distance. The signal from one input line is multiplied with the temporally filtered signal from the other line, and two antisymmetric units of this kind are subtracted from each other with equal weights, leading to a fully opponent, and thus highly directionally selective EMD (Borst and Egelhaaf, 1989). The time constant of the first-order lowpass filter, which is the fundamental temporal model parameter, was set to 2 frame intervals $(80 \mathrm{~ms})$ for the present study. The time interval between successive image frames corresponded to 8 digital simulation steps; an increased temporal resolution was used in order to improve the accuracy in calculating the dynamic responses of the temporal filters.

Movie sequences were processed by two 2D-arrays of such EMDs (two sets of 450 x 185 correlators, one pair centred at each image pixel, oriented along the horizontal or along the vertical axis of the cartesian-coordinate images). The result is a twodimensional motion signal distribution, the 2DMD response, with a horizontal and vertical signal component for each image point, which we call a motion signal map (see figure 1D). In some cases this raw 2DMD output was temporally averaged (over 8 to 64 frames) before further analysis. To be able to plot such signal maps at high spatial resolution, usually a two-dimensional colour code is used to represent the direction and the magnitude of local motion detector responses in terms of hue and saturation (Zeil and Zanker, 1997). For purposes of black and white reproduction, in the present study the horizontal and vertical motion components are plotted separately in grey-level maps. In the vertical components map a change from black through medium grey to white indicates a change from downwards motion through standstill to upwards motion (see figure 2A, D). Correspondingly, in the horizontal component map regions of motion to the left, no motion signal, and motion to the right correspond to black, medium grey and white regions, respectively (see figure $2 \mathrm{~B}, \mathrm{E}$ ). 


\section{$3 \quad$ Analysing motion signal maps}

The 2DMD response for a simple forward translation is shown in figure 2, comparing the output for a single displacement step (A-C) to the average of 16 consecutive steps (D-F). The vertical components of the motion signal maps (figure 2A \& D) are characterised by predominantly downwards directions in the lower half of the central image region, i.e. in the parts of the map that correspond to the field of view below the horizon looking forwards, and faint upwards signals in the upper half of the frontal region. The general direction is inverted for the image regions corresponding to the rear field of view, close to the left and right image borders. This pattern reflects the vertical expansion components from the expanding and contracting poles of the flowfield, which in the case of forward translation should be located in the centre and in the left/right border regions of the panoramic image. The horizontal components of the motion signal maps (figure $2 \mathrm{~B} \& \mathrm{E}$ ) are different in the left and the right half of the panoramic image, the former being dominated by dark spots that indicate leftward motion components, and the latter being dominated by bright spots that correspond to rightward motion. This distribution of local motion signals is typical for a forward translation of the camera which produces an expanding and contracting flowfield pole in the frontal and the rear field of view. Corresponding patterns of local motion signals, with different locations of the flowfield pole in the images, are found for translations in other directions (data not shown). Three peculiarities of the motion signal maps should be noted.

(a) The distribution of local motion signals is noisy and sparse. To emphasize the overall structure of the signal maps, a nonlinear grey-scale is used in figure 2 which saturates at about $10 \%$ of the local response maximum, thus leading to an underestimation of the sparseness of this map. To quantify the density and coherence of local motion signals, we focus on two typical regions extending approximately $15^{\circ}$ by $15^{\circ}$ (indicated by small frames in figure $2 \mathrm{~A}$ and B) just above and below the horizon in the left lateral field of view ( $-90^{\circ}$ azimuth), where the flowfield should be characterised by strong, coherent motion in horizontal direction $\left(+/-180^{\circ}\right)$. The actual sparseness of the motion map in these test regions is demonstrated by the fact that on average $93 \%$ of the signals are smaller than a tenth of the maximum motion signal present in the map, and $31 \%$ are smaller than a hundredth of the maximum signal. This result is not surprising because the density of the motion signal maps is determined by the strength of local motion detector responses, which depends on the abundance, contrast, and angular velocity of local contours. Far distant objects that only cause minute image displacements during translation, or a cloudless blue sky, for instance, do not elicit any significant motion detector response. Most importantly, the image region around the flowfield pole is characterised by a "hole", devoid of any clear motion signals. Furthermore, the directional noise level in the motion maps is reflected by the fact that the direction of no more than $17 \%$ of the motion signals in the test regions is found within a range of $30^{\circ}\left(47 \%\right.$ within a range of $\left.90^{\circ}\right)$ around the expected direction of $+/-180^{\circ}$. The fact that directions within small regions vary considerably can be attributed to fluctuations inherent to the EMD output (Reichardt and Egelhaaf, 1988) and to variations of local contour orientations which affect the direction of motion detected for these contours (Hildreth and Koch, 1987). 


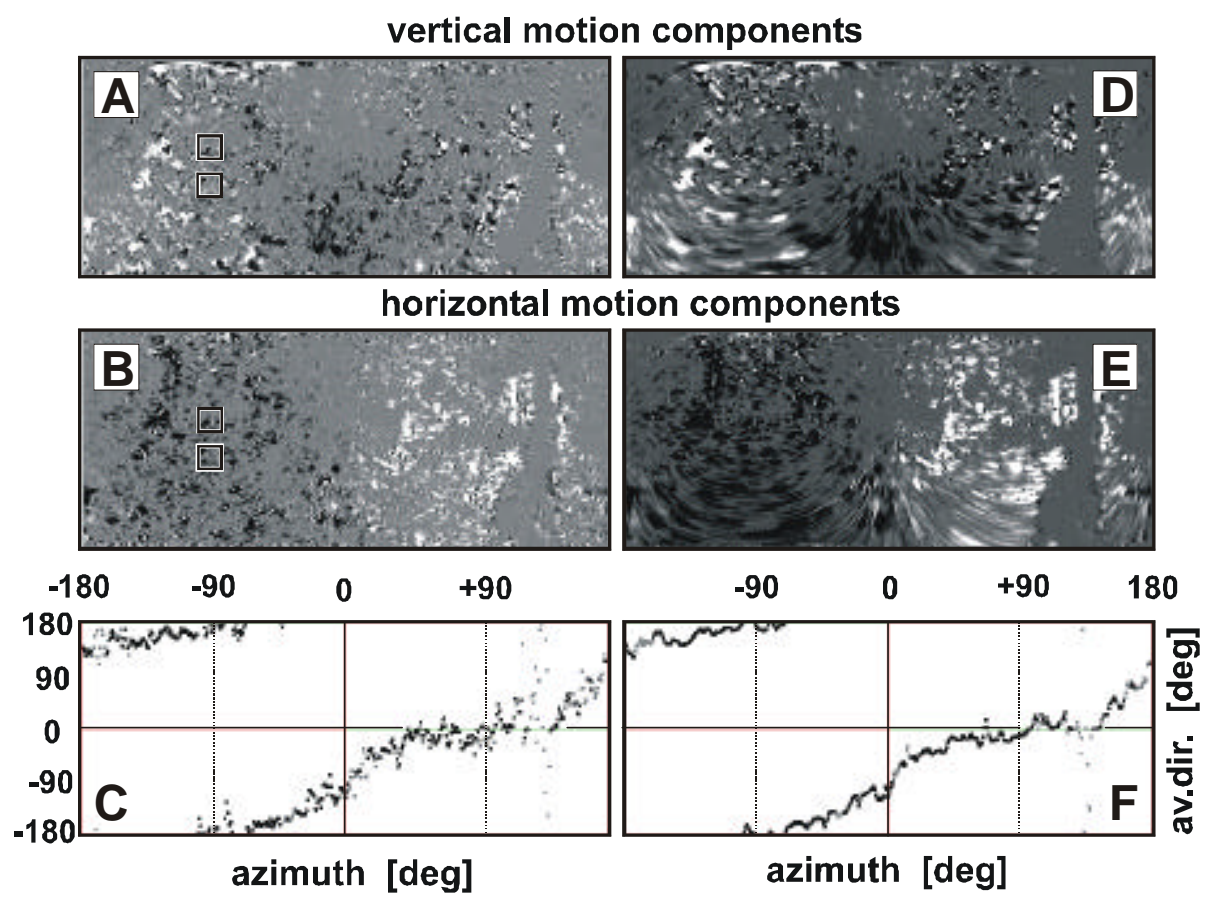

Fig. 2. 2DMD output. Motion signal maps (vertical motion response components A, D, up: white, no motion: medium grey, down: black; horizontal components B, E, right: white, no motion: medium grey, left: black) and average direction profiles (C, F) for a panoramic image sequence recorded during forward translation; A-C: response from a single displacement step; DF: temporal average from 16 consecutive displacement steps.

(b) There is a general tendency that motion signals are denser and stronger in the lower than in the upper image regions, because the camera is moving close to the ground where nearby contours generate comparatively large image motion components. On the other hand, objects above the horizon are much further away and therefore generate much smaller image angular displacements during translation. To quantify this effect, we compared the distribution of motion signal strengths in two test regions shown in figure $2 \mathrm{~A}$ and $\mathrm{B}$ with each other. Whereas $17 \%$ of the motion signals in the test region below the horizon are smaller than a hundredth of the maximum signal, $31 \%$ of the motion signals in the test region above the horizon fall below this limit.

(c) In the temporal averages of several 2DMD response frames (figure 2D \& E) motion signals are aligned along "motion streaks", which reflect the trajectories of image contrast elements during the averaging interval. These small, oriented streaks, contain independent information about the structure of the motion signal map - the radial patterns in the front and the rear image regions provide a clear indication of the flowfield poles. Recent psychophysical experiments suggest that humans actually are able to use the orientation information of temporally blurred moving objects for motion processing (Geisler, 1999, Burr et al., ECVP 2002). It should be noted that such a 
combination of orientation and motion information goes beyond the interaction between collinear direction selective motion detectors along motion trajectories (Watamaniuk and McKee, 1998), which would correspond to the Gestalt law of "common fate". The motion signal maps presented here suggest that a combination of information across stimulus modalities could indeed be very powerful for the extraction of flowfield parameters.

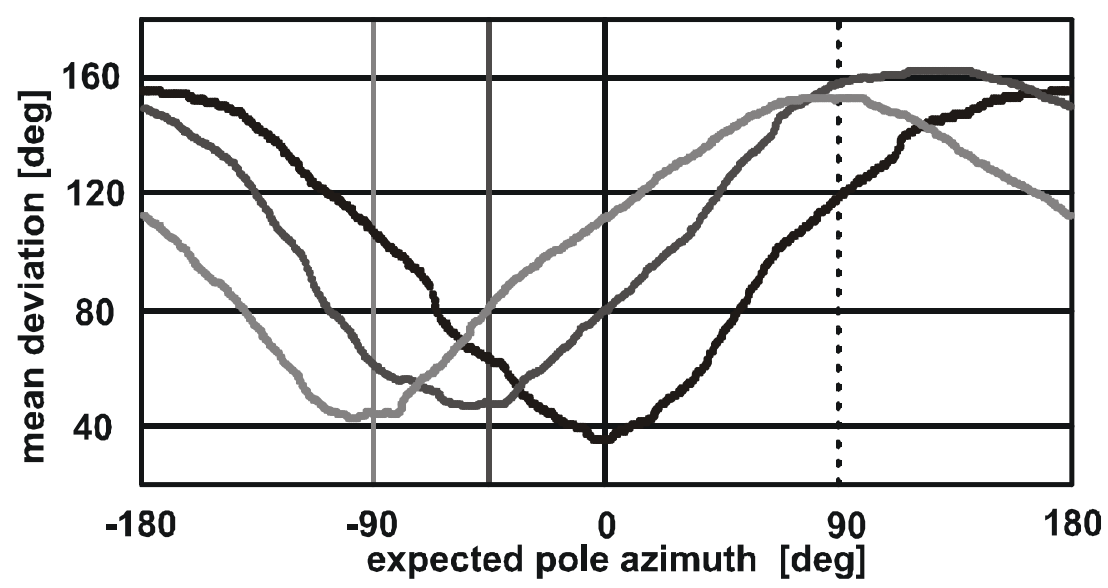

Fig. 3. Estimating the location of the flowfield pole. Average deviation between the expected direction profile function and the actual 2DMD output data plotted as function of the azimuth location of the expected flowfield pole for forward (black data points), sideways (light grey) and oblique (dark grey) translation. Minimum deviation indicate the best fit estimate of the flowfield pole, which are generally very close to the veridical values (indicated by vertical lines).

In a next step we condensed the motion signal maps to horizontal direction profiles by averaging EMD outputs along image columns, i.e. for a given horizontal position in the panoramic image. In these profiles, the direction of the average motion vector is plotted as a function of azimuth (figure $2 \mathrm{C} \& \mathrm{~F}$ ). The length of the average vector, i.e. motion signal strength which is determined by the average contrast and the inverse of the direction variance at a given azimuth, is represented by the greylevel of a given data point in the direction profiles (values above/below the overall average are plotted in black/grey). The response profiles capture some significant aspects of the motion signal maps, and in particular give a good indication of the location of the flowfield poles. In the lateral image regions the average motion direction is horizontal, close to $+/-180$ deg on the left side (around -90 deg azimuth) and close to 0 deg on the right side, clearly reflecting the homogenous image flow in the field of view perpendicular to the direction of egomotion. In the frontal and rear image regions, the profiles are characterised by a transition between the two horizontal motion directions. The vertical average direction at the locations of the flowfield pole, downwards (about -90 $\mathrm{deg}$ ) in the frontal region (around 0 deg azimuth) and upwards (about $+90 \mathrm{deg}$ ) in the rear image region (around $+/-180 \mathrm{deg}$ ), confirm the earlier observation that the signal maps are dominated by the regions below the horizon (where expansion and contrac- 
tion centred around the pole assumes these directions). The noise on the direction profiles is considerably reduced by temporal integration (compare figure $2 \mathrm{C} \mathrm{\&} \mathrm{F}$ ), and a similar effect is to be expected for spatial pooling. In summary, the directional profiles offer a fair account of the natural flowfield structure, as far as the location of poles is concerned, which indicate the direction of egomotion.

In order to analyse the direction profiles further, we fitted a simple mathematical function to the data which reflects the expectation of a basic directional profile. Because this technique intends to assess the quality of the information contained in the direction profiles and is not meant to suggest a particular mechanism dealing with optic flowfields, we made no attempt to specify anything like an optimal expectation function, similar to those used in matched filter approaches of estimating egomotion parameters (see, for instance, Franz and Krapp, 2000). Instead, we started from the idealised situation that in a homogenous flowfield generated by horizontal egomotion vertical velocity components would cancel each other, leading to a rectangular direction profile with horizontal motion to the left $(+/-180 \mathrm{deg})$ and to the right $(0 \mathrm{deg})$ on the left and right side of the expansion pole, respectively. This simple expectation function was shifted relative to the direction profiles by variation of the azimuth location of the assumed flowfield pole ('expected pole'), and the root mean square of the deviation between expectation and data was calculated. The deviation should reach a minimum when the expected pole is closest to the direction of egomotion. The mean deviation between data and expectation function is plotted in figure 3 as function of expected pole azimuth for three individual 2DMD output frames from three different movie sequences. Forwards, leftwards, and oblique translation should lead to a minimum at $0,-45$, and -90 deg of azimuth, respectively (indicated by vertical lines). It is obvious that the minima are very close to the respective veridical locations, with deviations of $-0.8,8.4$, and $6.2 \mathrm{deg}$ for the three cases tested. The mean deviation curves are virtually indistinguishable for different 2DMD output frames of an individual sequence, and no further improvement is found when motion signal maps are generated by averaging several frames (data not shown). This result suggests that the instantaneous information contained in the motion signals generated by a small camera displacement is comparable to the information that results from pooling signals over time while contours move along a trajectory, quite in contrast to the qualitative impression that averaged motion signal maps provide a much clearer flowfield structure (compare figure 2A-C with 2D-F). Elaborate mechanisms to estimate egomotion parameters (Perrone, 1992; Franz and Krapp, 2000) may thus not always be necessary, because under certain circumstances fast approximate procedures may be reliable and accurate enough (see also Dahmen et al. 1997).

\section{Conclusions}

The structure of optic flowfields that is influenced by the type of observer locomotion and by the structure of the environment, but also by neural processing strategies such as the basic motion detection mechanism or spatiotemporal pooling. The flowfield information available under realistic operating conditions was studied here by moving a panoramic imaging through natural environments and using the recorded image se- 
quences as input to a biologically plausible motion detector network. The resulting motion signal maps have several interesting properties, which reflect specific environmental and computational constraints of optic flow processing. Firstly, motion signals are sparsely distributed across the visual field, even in densely contourpopulated natural scenes. This property of motion signal maps would be predicted by motion detection theory, because the contrast dependence of the EMD response leads to irregular patterns of image regions at which the motion is clearly detectable. Secondly, local variation of motion direction is considerable, because the EMD output does not necessarily reflect the physical motion direction (Reichardt and Schlögl, 1988), a constraint which is well known in human psychophysics as the "aperture problem" (Adelson and Movshon, 1982; Nakayama and Silverman, 1988).

Nevertheless, panoramic motion signal maps contain sufficient information to estimate the direction of egomotion rather accurately, even with very simple estimation algorithms. This suggests that the inherent richness of flowfields in natural scenes might allow for fast, robust and simple mechanisms for sensing egomotion. We already know that under idealised conditions, the exact estimation of egomotion parameters requires only a small number of motion signals (Koenderink and Van Doorn, 1987; Dahmen et al., 2001), and we show here that this is also true for natural operating conditions. Future research will need to test whether this conclusion still holds for a wider range of movements, including mixtures of translation and rotation, and a variety of environments, including those with highly non-uniform distribution of objects. Conventional models of optic flow processing, which are able to separate translation and rotation components and control the direction of heading (Hildreth, 1984; Heeger, 1987; Perrone, 1992), usually rely on the assumption that dense and coherent motion signal maps are available. It will be essential to see how well such models deal with natural motion signal distributions and how the information content of natural flow fields is affected by the restricted field of view of many animals. In this context, it may become necessary to re-evaluate strategies to improve the spatial structure of the motion signal maps, such as local spatial pooling, temporal integration, or the extraction of true local velocity estimates (Verri et al., 1992) and to recognise the behavioural, sensory and neural adaptations animals are known to have evolved in response to the specific statistics of distance, contrast and contour orientation in their particular visual habitats (Nalbach and Nalbach, 1987; Nalbach, 1990; O'Carroll et al., 1997) (reviewed in Eckert and Zeil, 2001). It will be furthermore interesting to assess the value of additional information sources such as orientation cues provided by motion streaks.

To understand the evolution of behavioural and neural strategies of information processing and their adaptive quality, requires a more quantitative assessment of the natural operating conditions in which this evolution took and continues to take place. We hope to extend our preliminary study to a systematic and quantitative analysis of the motion signal distributions which are generated by different styles of locomotion in different environments. 


\section{Acknowledgements}

We are grateful to M. Franz and W. Junger for their support during the design and testing of the gantry and the recording of some image sequences, and to J. Chahl,, M. Hofmann, and M. V. Srinivasan for many discussions throughout the project. We acknowledge financial support from DSTO, HFSP and Wellcome Trust.

\section{References}

Adelson EH, Bergen JR. 1985. Spatiotemporal energy models for the perception of motion. Journal of the Optical Society of America A 2:284-299.

Adelson EH, Movshon JA. 1982. Phenomenal coherence of moving visual patterns. Nature 300:523-525.

Borst A, Egelhaaf M. 1989. Principles of visual motion detection. Trends in Neuroscience 12:297-306.

Chahl JS, Srinivasan MV. 1997. Reflective surfaces for panoramic imaging. Applied Optics 36:8275-8285.

Dahmen HJ, Franz MO, Krapp HG. 2001. Extracting egomotion from optic flow: limits of accuracy and neural matched filters. In: Zanker JM, Zeil J, Editors. Motion Vision - Computational, Neural, and Ecological Constraints. Berlin Heidelberg New York: Springer. p 143168.

Dahmen HJ, Wüst RM, Zeil J. 1997. Extracting egomotion parameters from optic flow: principal limits for animals and machines. In: Srinivasan MV, Venkatesh S, Editors. From living eyes to seeing machines. Oxford: Oxford University Press. p 174-198.

Eckert MP, Zeil J. 2001. Towards an ecology of motion vision. In: Zanker JM, Zeil J, Editors. Motion Vision: Computational, neural and ecological constraints. Berlin Heidelberg New York: Springer Verlag. p 333-369.

Franz MO, Krapp HG. 2000. Wide-field, motion-sensitive neurons and matched filters for optic flow fields. Biological Cybernetics 83:185-197.

Frost BJ, Wylie DR, Wang Y-C. 1990. The processing of object and self-motion in the tectofugal and accesory optic pathways of birds. Vision Research 30:1677-1688.

Geisler WS. 1999. Motion streaks provide a spatial code for motion direction. Nature 400:6569.

Gibson JJ. 1950. The perception of the visual world. Cambridge, MA: The Riverside Press.

Hausen K, Egelhaaf M. 1989. Neural Mechanisms of Visual Course Control in Insects. In: Stavenga DG, Hardie RC, Editors. Facets of Vision. Berlin Heidelberg: Springer Verlag. p 391-424.

Heeger DJ. 1987. Model for the extraction of image flow. Journal of the Optical Society of America A 4:1455-1471.

Hildreth E-C. 1984. The computation of the velocity field. Procedings of the Royal Society London B 221:189-220.

Hildreth E-C, Koch C. 1987. The analysis of visual motion: From computational theory to neuronal mechanisms. Annual Review of Neuroscience 10:477-533.

Johnston A, McOwan PW, Benton CP. 1999. Robust velocity computation from a biologically motivated model of motion perception. Procedings of the Royal Society London B 266:509518.

Koenderink JJ, Van Doorn AJ. 1987. Facts on Optic Flow. Biological Cybernetics 56:247-254.

Krapp HG, Hengstenberg R. 1996. Estimation of self-motion by optic flow processing in single visual interneurons. Nature 384:463-466. 
Longuet-Higgins HC, Prazdny K. 1980. The interpretation of a moving retinal image. Procedings of the Royal Society London B 208:385-397.

Nakayama K, Silverman GH. 1988. The aperture problem - II- Spatial integration of velocity information along contours. Vision Research 28:747-753.

Nalbach H-O. 1990. Multisensory control of eyestalk orientation in decapod crustaceans: an ecological approach. Journal of Crustacean Biology 10:382-399.

Nalbach H-O, Nalbach G. 1987. Distribution of optokinetic sensitivity over the eye of crabs: its relation to habitat and possible role in flow-field analysis. Journal of Comparative Physiology A 160:127-135.

O'Carroll D, Laughlin SB, Bidwell NJ, Harris SJ. 1997. Spatio-Temporal Properties of Motion Detectors Matched to Low Image Velocities in Hovering Insects. Vision Research 37:34273439.

Orban GA, Lagae L, Verri A, Raiguel S, Xiao D, Maes H, Torre V. 1992. First-order analysis of optical flow in monkey brain. Proceedings of the National Academy of Sciences USA 89:2595-2599.

Patzwahl DR, Zanker JM. 2000. Mechanisms for human motion perception: combining evidence from evoked potentials, behavioural performance and computational modelling. European Journal of Neuroscience 12:273-282.

Perrone JA. 1992. Model for the computation of self-motion in biological systems. Journal of the Optical Society of America A 9:177-194.

Reichardt W. 1987. Evaluation of optical motion information by movement detectors. Journal of Comparative Physiology A161:533-547.

Reichardt W, Egelhaaf M. 1988. Properties of Individual Movement Detectors as Derived from Behavioural Experiments on the Visual System of the Fly. Biological Cybernetics 58:287294.

Reichardt W, Schlögl RW. 1988. A two dimensional field theory for motion computation. First order approximation; translatory motion of rigid patterns. Biological Cybernetics 60:23-35.

Srinivasan MV. 1990. Generalized Gradient Schemes for the Measurement of TwoDimensional Image Motion. Biological Cybernetics 63:421-431.

Van Santen JPH, Sperling G. 1985. Elaborated Reichardt detectors. Journal of the Optical Society of America A 2:300-321.

Verri A, Straforini M, Torre V. 1992. Computational aspects of motion perception in natural and artificial vision systems. Philosophical Transactions of the Royal Society [Biology] 337:429-443.

Watamaniuk SNJ, McKee SP. 1998. Simltaneous encoding of direction at a local and global scale. Perception and Psychophysics 60:191-200.

Watson AB, Ahumada AJ. 1985. Model of human visual-motion sensing. Journal of the Optical Society of America A2:322-342.

Zanker JM. 2001. Combining Local Motion Signals: A Computational Study of Segmentation and Transparency. In: Zanker JM, Zeil J, Editors. Motion Vision: Computational, Neural and Ecological Constraints. Berlin Heidelberg New York: Springer.

Zanker JM, Hofmann MI, Zeil J. 1997. A two-dimensional motion detector model (2DMD) responding to artificial and natural image sequences. Investigative Ophthalmology and Visual Science 38:S 936.

Zeil J, Voss R, Zanker JM. 1998. A View from the Cockpit of a Learning Wasp. In: Elsner N, Wehner R, Editors. Gottingen Neurobiology Report 1998. Stuttgart: Georg Thieme Verlag. p 140.

Zeil J, Zanker JM. 1997. A Glimpse into Crabworld. Vision Research 37:3417-3426. 